\title{
Knockdown of LETM1 inhibits proliferation and metastasis of human renal cell carcinoma cells
}

\author{
JIE XU ${ }^{1,2}$, BISHENG HUANG ${ }^{1}$, SAIYANG LI $^{3}$, XIAOLU ZHANG ${ }^{1}$, TIANCHENG XIE ${ }^{1}$ and YUNFEI XU ${ }^{1,3}$ \\ ${ }^{1}$ Department of Urology, Shanghai Tenth People's Hospital, Tongji University School of Medicine, \\ Shanghai 200072; ${ }^{2}$ Department of Urology, Pudong New Area People's Hospital, Shanghai 201299; \\ ${ }^{3}$ Department of First Clinical Medical College, Nanjing Medical University, Nanjing, Jiangsu 210029, P.R. China
}

Received December 8, 2017; Accepted August 30, 2018

DOI: $10.3892 / \mathrm{ol} .2018 .9449$

\begin{abstract}
The leucine zipper-EF-hand containing transmembrane protein 1 (LETM1) has been reported to serve an important role in a number of human malignancies and is correlated with poor prognosis. However, little is known about the role of LETM1 in renal cell carcinoma (RCC). In the present study, the expression levels of LETM1 were investigated in RCC cell lines (Caki-1, 786-O, OS-RC-2, A498 and ACHN) and the HK-2 normal human renal tubular epithelial cell line. Short interfering RNA (siRNA) was used to knock down the expression of LETM1 in 786-O and A498 cells. The results indicated that the constitutive expression of LETM1 was notably upregulated in RCC cell lines. Knockdown of LETM1 significantly decreased cell proliferation, migration and invasion. Mechanistically, it was revealed that the knockdown of LETM1 expression sharply downregulated the protein expression of $\beta$-Catenin, Cyclin D1 and c-Myc in 786-O and A498 cells. In conclusion, these results suggest that knockdown of LETM1 exhibits tumor suppressive effects, at least in part by controlling the downstream Wnt/ß-Catenin signaling pathway. Therefore, LETM1 may act as a novel therapeutic target for the treatment of RCC.
\end{abstract}

\section{Introduction}

There are approximately 63,990 new cases of kidney cancer and an estimated 14,400 cancer associated deaths in the United States in 2017 (1). Renal cell carcinoma (RCC) represents one of the most common type of kidney cancer, accounting for $85-90 \%$ of renal malignancies (2). Nephrectomy and nephron-sparing surgery are effective clinical management for

Correspondence to: Professor Yunfei Xu, Department of Urology, Shanghai Tenth People's Hospital, Tongji University School of Medicine, 301 Yanchang Road, Shanghai 200072, P.R. China

E-mail: xuyunfeibb@sina.com

Key words: renal cell carcinoma, leucine zipper-EF-hand containing transmembrane protein 1 , short interfering RNA, proliferation, metastasis localized RCC. However, Only $30 \%$ of patients are diagnosed on the basis of symptoms, and up to $17 \%$ of all RCC patients present with distant metastases at the time of diagnosis (3). Metastatic RCC is highly resistant to current available therapies including chemotherapy, immunotherapy using interferon-a (IFN-a) and targeted therapy using tyrosine kinase inhibitors, while the underlying mechanisms of how these metastasis occur are largely uncharacterized. It is needed to identify novel molecular targets for the better management of RCC.

Leucine zipper-EF-hand containing transmembrane protein 1 (LETM1), first reported in 1999 and is deleted in nearly all Wolf-Hirschhorn syndrome (WHS) patients (4), is a mitochondrial inner membrane protein and plays an important role in mitochondrial ATP production and biogenesis $(5,6)$. Piao et al (6), first reported the role of LETM1 in tumorigenesis in 2009, they showed that dysregulation of LETM1 is a key feature of tumorigenesis by altering cancerous metabolic reactions. Recently, several studies reported that high expression of LETM1 predicts poor prognosis (7) and associated with the clinicopathological parameters of human cancers (8-10). However, the role of LETM1 in RCC has not yet been determined.

In the present study, we demonstrated that LETM1 overexpression significantly correlated with poor prognosis of RCC patients and knockdown of LETM1 markedly decreased the proliferation, migration and invasion of RCC cells.

\section{Materials and methods}

Cell lines and cultures. Human RCC cell lines (Caki-1, 786-O, OS-RC-2, A498 and ACHN) and human normal renal tubular epithelial cell line HK-2 were obtained from the cell bank of type culture collection of the Chinese Academy of Sciences (Shanghai, China). Caki-1, A498 and ACHN were cultured in Dulbecco's Modified Eagle's Medium (DMEM; Gibco; Thermo Fisher Scientific, Inc., Waltham, MA, USA). 786-O and OS-RC-2 cells were maintained in RPMI-1640 medium (Gibco; Thermo Fisher Scientific, Inc.). HK-2 cells were cultured in DMEM/F12 (Sigma-Aldrich; Merck KGaA, Darmstadt, Germany) containing $5 \mathrm{mM}$ glucose. These media were supplemented with $10 \%$ fetal bovine serum (FBS; Gibco; Thermo Fisher Scientific, Inc.) and $1 \%$ penicillin/streptomycin (HyClone; GE Healthcare Life Sciences, Logan, UT, USA). 
All cells were incubated in a humidified incubator with $5 \%$ $\mathrm{CO}_{2}$ at $37^{\circ} \mathrm{C}$.

Xena public datahubs.UCSCXenabrowser(https://xenabrowser. net/heatmap/) was used to obtain the Cancer Genome Atlas (TCGA) Kidney Clear Cell Carcinoma data and to evaluate the overall survival.

Cell transfection. RCC cells 786-O and A498 were transfected with double-stranded short interfering RNA (siRNA) (Shanghai GenePharma Co., Ltd., Shanghai, China) with Lipofectamine 2000 (Invitrogen; Thermo Fisher Scientific, Inc.) according to the manufacturer's instructions. At $72 \mathrm{~h}$ after transfection, the gene silencing effect was measured using western blot analysis. The target sequence of LETM1 siRNA is: 5'-CCACAGAAUCGUGUCUGGAUCCACA-3' and the sequence of negative control siRNA is: 5'-CGAGCAGAGACT CTAACATTCTCGC-3'.

Cell proliferation assay. Cell proliferation was assessed using Cell Counting Kit-8 (CCK-8; Dojindo Molecular Technologies Inc., Kumamoto, Japan). Briefly, 1,000 cells/100 $\mu \mathrm{l} /$ well in 96-well plate were seed, and then $10 \mu \mathrm{l}$ of CCK-8 was added into each well at indicated time-points (24, 48, 72 and $96 \mathrm{~h})$ and incubation followed for $2 \mathrm{~h}$ at $37^{\circ} \mathrm{C}$. The absorbance at $450 \mathrm{~nm}$ was detected on a microplate spectrophotometer (BioTek Instruments, Inc., Winooski, VT, USA).

Colony formation assay. For colony forming assay, 1,000 cells were seeded in six-well plates and cultured at $37^{\circ} \mathrm{C}$ in a humidified incubator. After two-weeks' incubation, the cells were fixed with $4 \%$ paraformaldehyde, then stained with $0.5 \%$ gentian violet and observed under a digital camera.

Transwell migration and invasion assays. Cell migration and invasion assays were performed using transwell chambers (BD Biosciences, San Jose, CA, USA). For the invasion assay, chambers were precoated with $25 \mu \mathrm{l}$ of Matrigel (BD Biosciences) at $37^{\circ} \mathrm{C}$ for $2 \mathrm{~h}$, whereas the chambers used for migration assay were not precoated with Matrigel. $5 \times 10^{4}$ transfected cells in $200 \mu \mathrm{l}$ serum-free medium were plated in the upper well of the, and $600 \mu \mathrm{l}$ of medium containing $10 \%$ FBS were added into the lower chambers as chemoattractant. After incubated at $37^{\circ} \mathrm{C}$ for $10 \mathrm{~h}$, cells on the upper surface of the insert were removed with a cotton swab, the cells migrating to the lower surface of the chamber were fixed with $95 \%$ ethanol for $20 \mathrm{~min}$, stained with $0.1 \%$ crystal violet solution for $20 \mathrm{~min}$. The migrated cells were counted under using light microscope (Olympus Corporation, Tokyo, Japan).

Western blot analysis. Cells were washed with cold phosphate-buffered saline (PBS) and lysed in ice-cold RIPA buffer (Sigma-Aldrich; Merck KGaA) containing protease inhibitor. BCA protein assay kit (Beyotime Institute of Biotechnology, Haimen, China) was used to determine the concentration of total cellular protein. Then equal amounts of protein were loaded into $10 \%$ SDS-PAGE gel and after separation transferred to nitrocellulose membranes, the membranes were blocked in $5 \%$ non-fat milk for one hour and then incubated with primary antibodies: Mouse anti-LETM1 (sc-271234, 1:500; Santa Cruz
Biotechnology, Inc., Dallas, TX, USA), mouse anti- $\beta$-Actin (sc-130300, 1:2,000; Santa Cruz Biotechnology, Inc.), rabbit anti- $\beta$-Catenin (no. 8480, 1:1,000; Cell Signaling Technology), rabbit anti-Cyclin D1 (ab134175, 1:2,000; Abcam, Cambridge, MA, USA), and rabbit anti-c-Myc (no. 5605, 1:2,000; Cell Signaling Technology) overnight at $4^{\circ} \mathrm{C}$. After washing with PBST three times, the membranes were incubated with the corresponding secondary antibodies (Goat anti-Rabbit IgG, 926-32211; LI-COR Biosciences, Lincoln, NE, USA; Goat anti-Mouse IgG, 926-32210; LI-COR Biosciences) at 1:2,000 dilution ratio at room temperature for one hour. The protein bands were visualized using the Odyssey two-color infrared laser imaging system (LI-COR Biosciences).

Statistical analysis. The SPSS v.16.0 statistical software (IBM SPSS, Armonk, NY, USA) was used for statistical analysis. All data are presented as means \pm standard deviation (SD) from at least three independent experiments. Survival analysis was evaluated by Log-rank test, other experiment results were calculated using Student's t-test or one-way analysis of variance followed by post-hoc LSD test. $\mathrm{P}<0.05$ was considered to indicate a statistically significant difference. The diagrams were drawn by GraphPad Prism v.5 software (GraphPad Software, Inc., La Jolla, CA, USA).

\section{Results}

LETM1 is upregulated in RCC cell lines and associated with poor prognosis. To explore the expression difference of LETM1 between RCC cell lines and human normal renal tubular epithelial cell line HK-2, western blot is performed. As shown in Fig. 1A, the protein level of LETM1 were significantly higher in RCC cell lines when compared with that in the normal human normal renal tubular epithelial cell line HK-2 cells. We further examined whether LETM1 expression correlated with outcome in RCC patients. Kaplan-Meier survival analysis and log-rank tests was performed using TCGA database. The Kaplan-Meier survival curve demonstrated that patients with high LETM1 expression had lower overall survival than those with low LETM1 expression (Fig. 1B). These results indicated that LETM1 was upregulated in RCC cell lines, we assumed that LETM1 might act as an oncogene in RCC.

Knockdown of LETM1 inhibits RCC cell proliferation. To further explore the role of LETM1 in RCC cells, 786-O and A498 cells were both transfected with si-LETM1 or si-NC respectively, the expression of LETM1 at protein level were detected at $72 \mathrm{~h}$ after transfection. We found that knockdown of LETM1 by si-LETM1 significantly decreased the LETM1 protein expression in both 786-O and A498 cells (Fig. 2A). Colony formation assay showed that knockdown of LETM1 obviously decreased the proliferation of 786-O and A498 cells, when compared with controls (Fig. 2B-D). Similar effects were observed in the CCK-8 proliferation assay (Fig. 2E and F). Taken together, knockdown of LETM1 markedly inhibited 786-O and A498 cell proliferation.

Knockdown of LETM1 inhibits RCC cell migration and invasion. As shown in Fig. 3A, the number of cells identified across the membrane in the negative control groups 

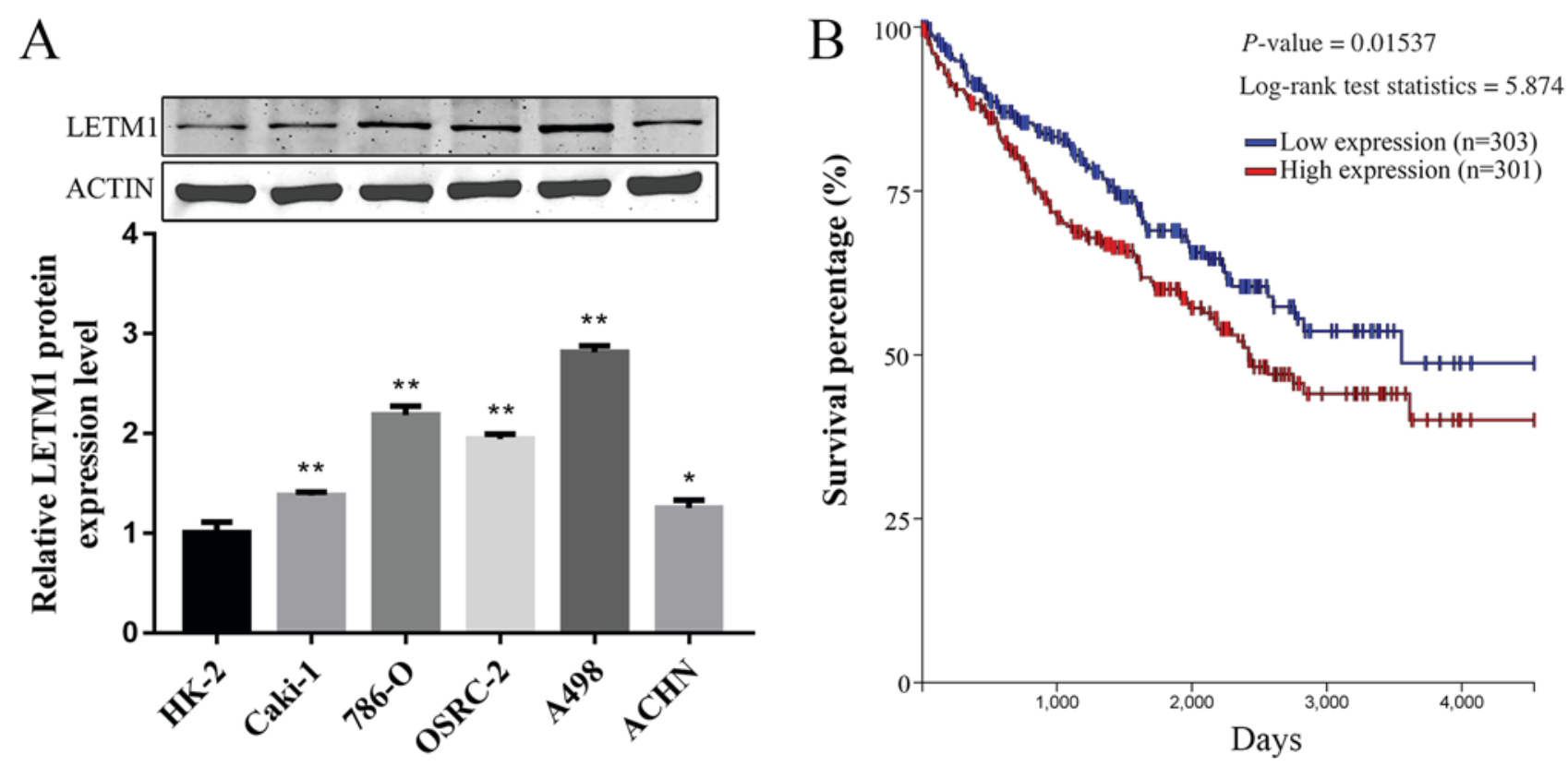

Figure 1. Constitutive expression of LETM1 in human RCC cell lines. (A) The constitutive protein expression of LETM1 was significantly upregulated in RCC cell lines compared with the HK-2 cell line. All experiments were performed in triplicate. ${ }^{*} \mathrm{P}<0.05,{ }^{* * *} \mathrm{P}<0.01$ vs. HK-2. (B) Patients with high LETM1 expression had a significantly reduced survival rate compared with patients with low LETM1 expression. LETM1, leucine zipper-EF-hand containing transmembrane protein 1; RCC, renal cell carcinoma.

were significantly higher than the number of cells across the membrane in the si-LETM1 group by using transwell chambers without Matrigel. The results showed that knockdown of LETM1 markedly inhibited the migration in 786-O and A498 cells. In addition, we explored the role of LETM1 on cell invasion of RCC cells using transwell chambers with Matrigel. As showed Fig. 3B, knockdown of LETM1 significantly suppressed the number of 786-O and A498 cells that invaded through Matrigel as compared with the negative control groups.

Knockdown of LETM1 inhibits the activation of Wnt/B-catenin pathway. To explore the underlying molecular mechanism of si-LETM1 inhibiting RCC cell proliferation and metastasis, the protein levels of $\beta$-Catenin, Cyclin D1, and c-Myc were evaluated using western blot in 786-O and A498 cells transfected with si-LETM1. As illustrated in Fig. 4A and B, knockdown of LETM1 significantly suppressed the protein expression of $\beta$-Catenin, Cyclin D1, and c-Myc in RCC cells compared with the negative control groups.

\section{Discussion}

RCC is a public health challenge worldwide as it is difficult to be detected in early stages. Emerging evidences indicate that investigating the underlying molecular mechanisms of RCC pathogenesis, and identifying novel treatments and targets for gene therapy are required to improve the prognosis of patients with RCC. Although great progress in diagnosis and treatment has been achieved in the past decades, several key factors and pathways have been identified to be responsible for renal tumorigenesis such as VHL, VEGF, NFKB, mTOR pathways (11-13). However, once metastatic disease develops, the prognosis for long-term survival of RCC patients is poor (14-16). In the present study, we first demonstrated that LETM1 is up-regulated in RCC cell lines, and suppression of LETM1 significantly suppressed cell proliferation, migration and invasion.

LETM1was first reported to be deleted in nearly all WHS patients (4). Recently, an increasing number of studies have shown that LETM1 is highly expressed in many kinds of human cancers and predicts poor prognosis $(7-10,17)$, this is consist with our results that up-regulated LETM1 expression was found in RCC cell lines and was related with poor prognosis of RCC patients. As for the molecular function of LETM1, Piao et al (6), found that LETM1 is overexpressed in various human cancers, and they demonstrated that metabolic alteration of cells may affect the progress of tumorigenesis. Doonan et al (18) found that knockdown of LETM1 caused accumulation of S-phase cells, and restoration of LETM1 could reverse S-phase accumulation, which was consistent with our previous study (8), indicating that suppression of LETM1 inhibited cell proliferation possibly by disrupting cell cycle distribution. However, Hwang et al (17) reported that LETM1 may acts a tumor suppressor in lung cancer by activation of AMPK activity and inhibition of Akt activity. It is still unclear how LETM1 affect tumorigenesis differ by tumor types. In this study, we demonstrated that suppression of LETM1 significantly suppressed cell proliferation, migration and invasion in vitro. However, more research is warranted in order to test the in vivo role of LETM1 in the tumor growth in nude mice.

Numerous studies revealed that the Wnt $/ \beta$-Catenin pathway is an important signaling pathway involved in the malignant progression of various tumors (19-22). Activated $\beta$-Catenin promoting transcription of target genes. Some target genes, including Cyclin D1 and c-Myc, are involved in cell proliferation and differentiation, cell migration, and tumor 

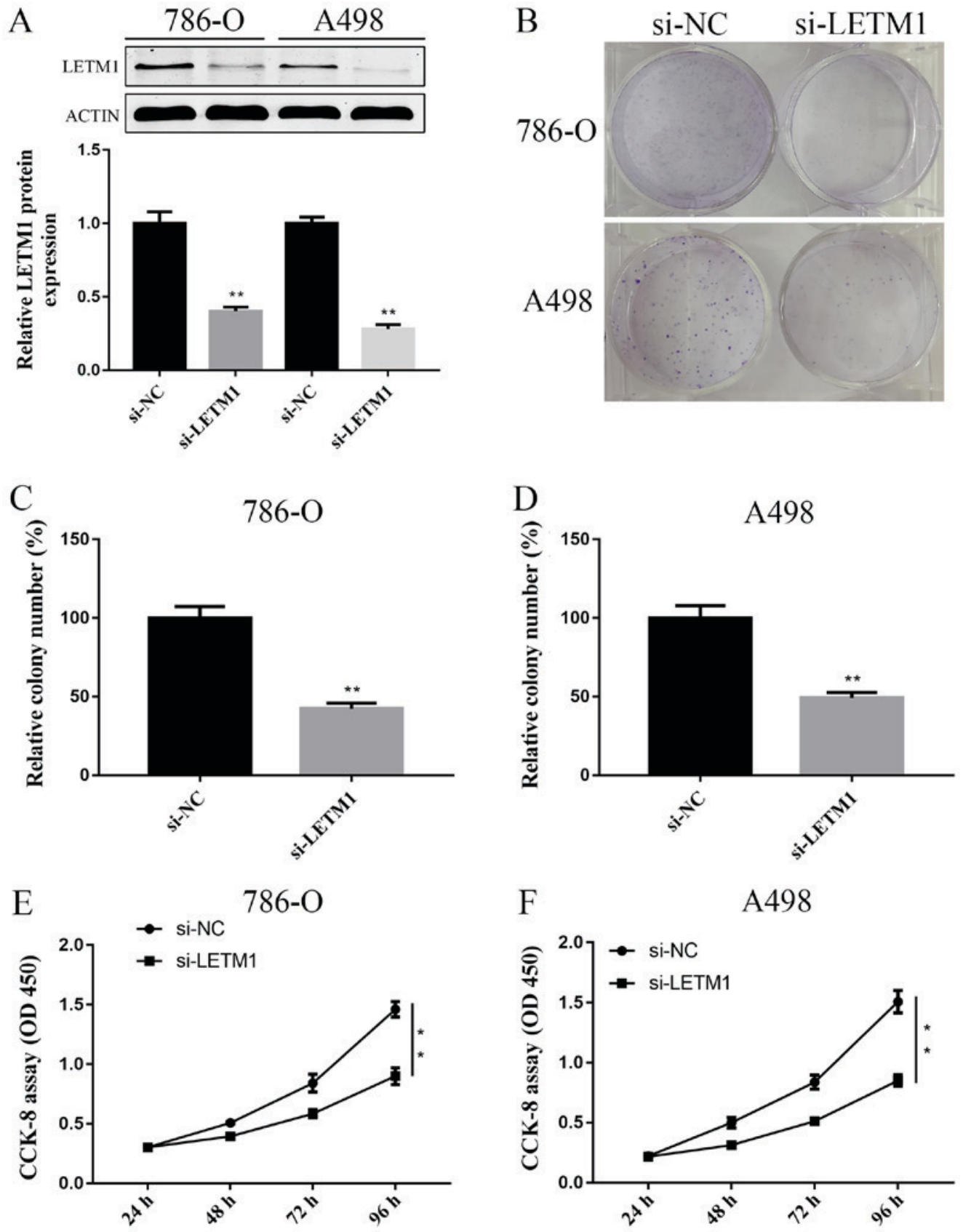

Figure 2. Knockdown of LETM1 inhibits cell proliferation in RCC cells. (A) LETM1 protein expression in 786-O and A498 cells was examined by western blotting. (B) Images of colony formation. The cell proliferation of (C) 786-O and (D) A498 cells was detected by a Colony formation assay. The cell proliferation of (E) 786-O and (F) A498 cells was detected by CCK-8 assay. All experiments were performed in triplicate. * P $<0.01$ vs. the control. NC, negative control; si, short interfering; LETM1, leucine zipper-EF-hand containing transmembrane protein 1; CCK, cell counting kit.

formation $(23,24)$. To the best of our knowledge, this is the first study that infers the relationship between LETM1 and Wnt/ $\beta$-Catenin pathway in RCC. In our study, we found that knockdown of LETM1 inhibited the expression of $\beta$-Catenin, Cyclin D1, and c-Myc in 786-O and A498 cells. These findings strongly suggest that LETM1 played an important role in tumor progression of RCC through the activation of Wnt/ $\beta$-Catenin signaling pathway.

In conclusion, the present study revealed that knockdown of LETM1 suppresses proliferation, migration and invasion of 786-O and A498 cells perhaps via suppressing the Wnt/ $\beta$-Catenin signaling pathway. Therefore, suppression of LETM1 may be a promising agent for treating RCC.

\section{Acknowledgements}

Not applicable.

\section{Funding}

This study was supported by research grants from the National Natural Science Foundation of China (grant no. 81671446).

\section{Availability of data and materials}

The analyzed datasets generated during the present study are available from the corresponding author on reasonable request. 
A
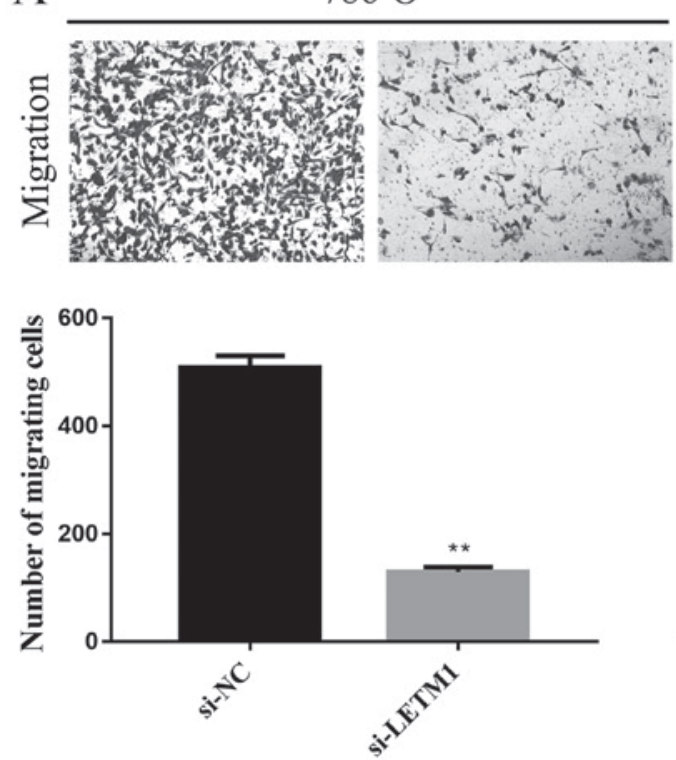

$\mathrm{B}$
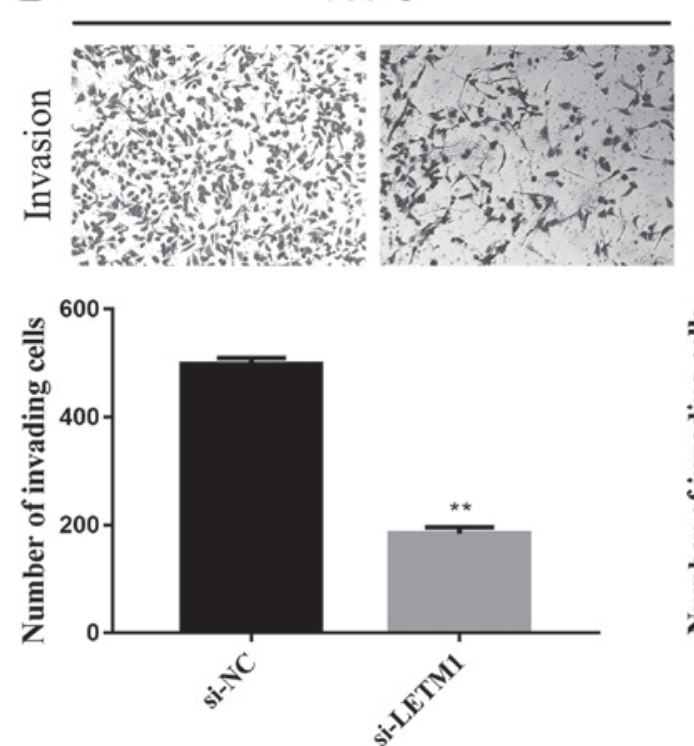

A498
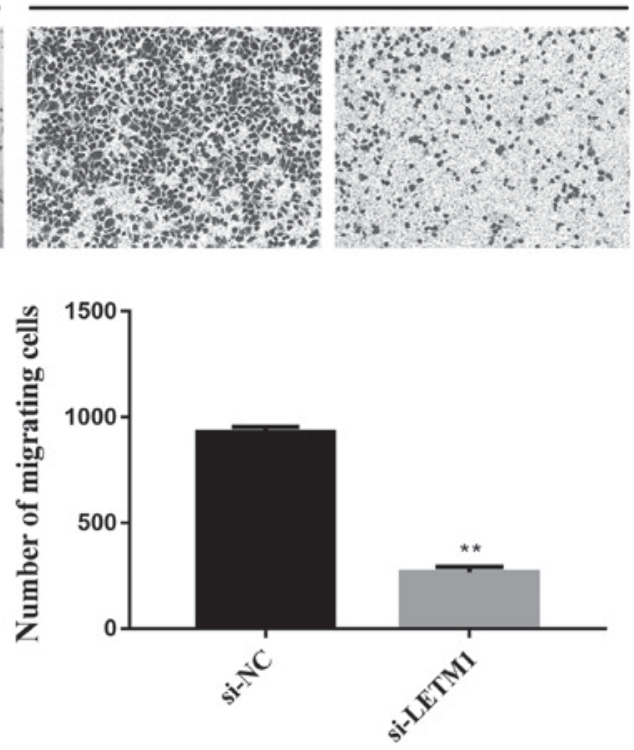

A498
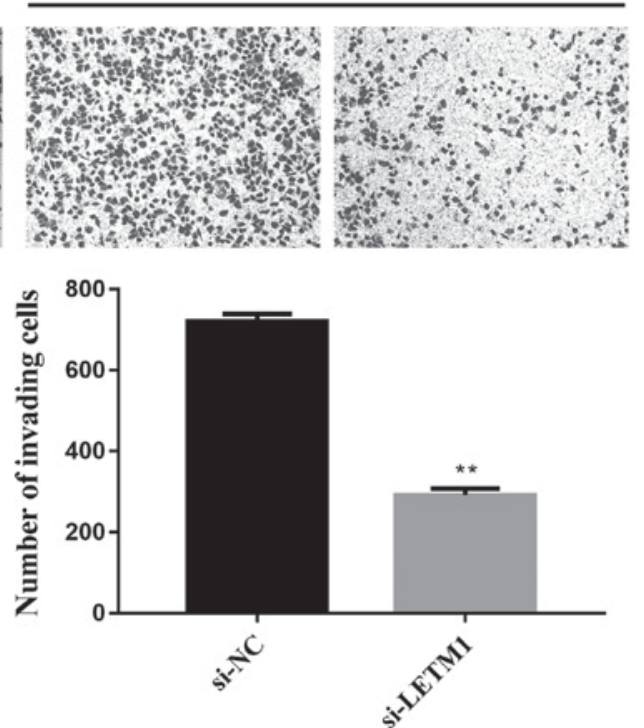

Figure 3. Downregulation of LETM1 inhibits the migration and invasion of RCC cells. After $48 \mathrm{~h}$ of transfection, a Transwell assay was used to evaluate the effects of si-LETM1 on (A) cell migration and (B) invasion (magnification, $\mathrm{x} 100$ ). All experiments were performed in triplicate. ${ }^{* * *} \mathrm{P}<0.01 \mathrm{vs.}$ the control. LETM1, leucine zipper-EF-hand containing transmembrane protein 1; RCC, renal cell carcinoma; NC, negative control; si, short interfering.
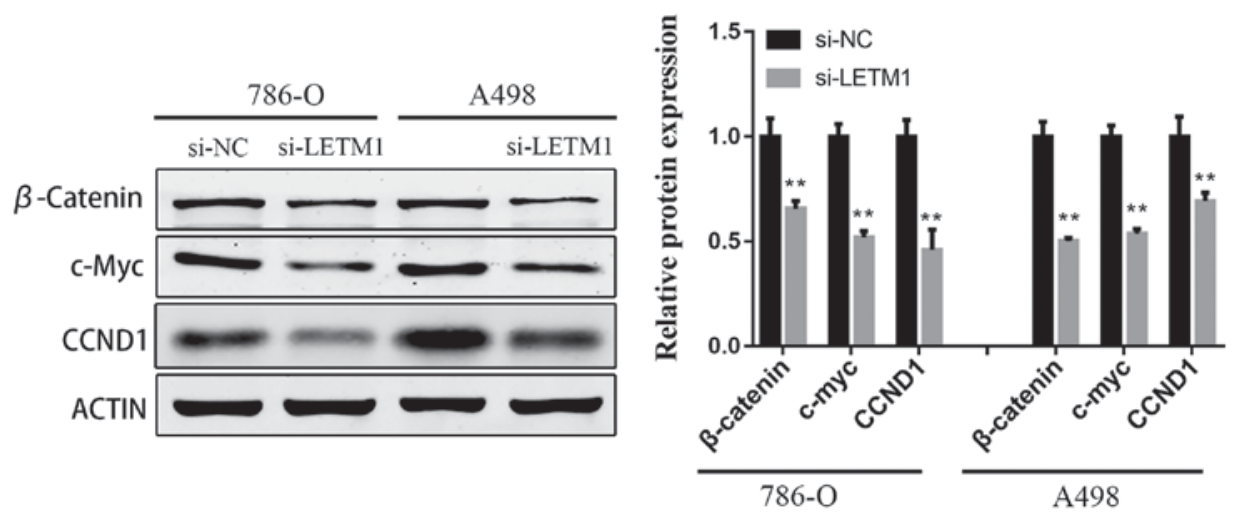

Figure 4. Downregulation of LETM1 inhibits the Wnt/B-Catenin signaling pathway in RCC cells. After $48 \mathrm{~h}$ of transfection, a western blot assay was used to detect the protein expression of $\beta$-Catenin, c-Myc and CCND1 in 786-O and A498 cells. All experiments were performed in triplicate. ${ }^{*} \mathrm{P}<0.05$, ${ }^{* * *} \mathrm{P}<0.01$ vs. the control. NC, negative control; si, short interfering; LETM1, leucine zipper-EF-hand containing transmembrane protein 1; RCC, renal cell carcinoma; CCND1, Cyclin D1. 


\section{Authors' contributions}

JX, BH and YX conceived and designed the experiments. JX and $\mathrm{BH}$ performed the experiments. SL, XZ and TX conducted the statistical analysis. JX and $\mathrm{BH}$ wrote the manuscript.

\section{Ethics approval and consent to participate}

Not applicable.

\section{Patient consent for publication}

Not applicable.

\section{Competing interests}

The authors declare that they have no competing interests.

\section{References}

1. Siegel RL, Miller KD and Jemal A: Cancer statistics, 2017. CA Cancer J Clin 67: 7-30, 2017.

2. Shuch B, Amin A, Armstrong AJ, Eble JN, Ficarra V, Lopez-Beltran A, Martignoni G, Rini BI and Kutikov A: Understanding pathologic variants of renal cell carcinoma: Distilling therapeutic opportunities from biologic complexity. Eur Urol 67: 85-97, 2015.

3. Capitanio U and Montorsi F: Renal cancer: Lancet 387: 894-906, 2016.

4. Endele S, Fuhry M,Pak SJ,Zabel BU and Winterpacht A: LETM1, a novel gene encoding a putative $\mathrm{EF}-\mathrm{hand} \mathrm{Ca}(2+)$-binding protein flanks the Wolf-Hirschhorn syndrome (WHS) critical region and is deleted in most WHS patients. Genomics 60: 218-225, 1999.

5. Nowikovsky K, Froschauer EM, Zsurka G, Samaj J, Reipert S, Kolisek M, Wiesenberger G and Schweyen RJ: The LETM1/YOL027 gene family encodes a factor of the mitochondrial $\mathrm{K}+$ homeostasis with a potential role in the Wolf-Hirschhorn syndrome. J Biol Chem 279 30307-30315, 2004.

6. Piao L, Li Y, Kim SJ, Byun HS, Huang SM, Hwang SK, Yang KJ, Park KA, Won M, Hong J, et al: Association of LETM1 and MRPL36 contributes to the regulation of mitochondrial ATP production and necrotic cell death. Cancer Res 69: 3397-3404, 2009.

7. Chen L, Yang Y, Liu S, Piao L, Zhang Y, Lin Z and Li Z: High expression of leucine zipper-EF-hand containing transmembrane protein 1 predicts poor prognosis in head and neck squamous cell carcinoma. Biomed Res Int 2014: 850316, 2014.

8. Huang B, Zhang J, Zhang X, Huang C, Hu G, Li S, Xie T, Liu M and Xu Y: Suppression of LETM1 by siRNA inhibits cell proliferation and invasion of bladder cancer cells. Oncol Rep 38: 2935-2940, 2017.
9. Li N, Zheng Y, Xuan C, Lin Z, Piao L and Liu S: LETM1 overexpression is correlated with the clinical features and survival outcome of breast cancer. Int J Clin Exp Pathol 8: 12893-12900, 2015.

10. Wang CA. Liu Q, Chen Y, Liu S, Xu J, Cui X, Zhang Y and Piao L: Clinical implication of leucine zipper/EF hand-containing transmembrane-1 overexpression in the prognosis of triple-negative breast cancer. Exp Mol Pathol 98: 254-259, 2015.

11. Rini BI, Campbell SC and Escudier B: Renal cell carcinoma. Lancet 373: 1119-1132, 2009.

12. Koul H, Huh JS, Rove KO, Crompton L, Koul S, Meacham RB and Kim FJ: Molecular aspects of renal cell carcinoma: A review. Am J Cancer Res 1: 240-254, 2011.

13. Rini BI: Vascular endothelial growth factor-targeted therapy in metastatic renal cell carcinoma. Cancer 115 (10 Suppl): S2306-S2312, 2009.

14. Motzer RJ, Bander NH and Nanus DM: Renal-cell carcinoma. N Engl J Med 335: 865-875, 1996.

15. Figlin RA: Renal cell carcinoma: Management of advanced disease. J Urol 161: 381-367, 1999.

16. Lanigan D: Prognostic factors in renal cell carcinoma. Br J Urol 75: 565-571, 1995.

17. Hwang SK, Piao L, Lim HT, Minai-Tehrani A, Yu KN, $\mathrm{Ha}$ YC, Chae CH, Lee KH, Beck GR, Park J and Cho MH: Suppression of lung tumorigenesis by leucine zipper/EF hand-containing transmembrane-1. PLoS One 5: pii: e12535, 2010.

18. Doonan PJ, Chandramoorthy HC, Hoffman NE, Zhang X, Cárdenas C, Shanmughapriya S, Rajan S, Vallem S, Chen X, Foskett JK, et al: LETM1-dependent mitochondrial Ca2+ flux modulates cellular bioenergetics and proliferation. FASEB J 28: 4936-4949, 2014.

19. Cojocaru E, Lozneanu L, Giuş̧ă SE, Căruntu ID and Danciu M: Renal carcinogenesis-insights into signaling pathways. Rom J Morphol Embryol 56: 15-19, 2015.

20. Wang $X$, Lu X, Geng Z, Yang G and Shi Y: LncRNA PTCSC3/miR-574-5p governs cell proliferation and migration of papillary thyroid carcinoma via Wnt/ß-catenin signaling. J Cell Biochem 118: 4745-4752, 2017.

21. Yuan H, Yu S, Cui Y, Men C, Yang D, Gao Z, Zhu Z and Wu J: Knockdown of mediator subunit Med19 suppresses bladder cancer cell proliferation and migration by downregulating Wnt/ $\beta$-catenin signalling pathway. J Cell Mol Med 21: 3254-3263, 2017.

22. Rahmani F, Avan A, Hashemy SI and Hassanian SM: Role of Wnt/ $\beta$-catenin signaling regulatory microRNAs in the pathogenesis of colorectal cancer. J Cell Physiol 233: 811-817, 2018.

23. Clevers $\mathrm{H}$ : Wnt/beta-catenin signaling in development and disease. Cell 127: 469-480, 2006.

24. Polakis P: The many ways of Wnt in cancer. Curr Opin Genet Dev 17: 45-51, 2007.

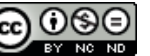

This work is licensed under a Creative Commons Attribution-NonCommercial-NoDerivatives 4.0 International (CC BY-NC-ND 4.0) License. 